\title{
Review
}

\section{Understanding and Managing Trauma-Induced Vestibular Deficits}

\author{
Art Mallinson $^{1,2}{ }^{\oplus}$, Raphaël Maire ${ }^{3}$, Christian Beyaert ${ }^{4,5,6}{ }^{\infty}$, Dominique Vibert ${ }^{7}$, \\ Laurent Coffinet ${ }^{4,8}$, Neil Longridge ${ }^{1}$, Robby Vanspauwen ${ }^{9}$, Georges Dumas ${ }^{4,10}{ }^{\circ}$, \\ Hannes Petersen ${ }^{11,12}$, Philippe Perrin ${ }^{4,6,8}$ (i) \\ 'Department of Surgery, Faculty of Medicine, University of British Columbia, Vancouver, Canada \\ ${ }^{2}$ Neuro-Otology Unit, Vancouver General Hospital Vancouver, Canada \\ ${ }^{3}$ Clinic of Otolaryngology, Head and Neck Surgery, Neurotology Unit, Lausanne University Hospital, Lausanne, Switzerland \\ ${ }^{4}$ Research Unit EA 3450 DevAH - Development, Adaptation and Handicap, University of Lorraine, Faculty of Medicine, Nancy, France \\ ${ }^{5}$ Regional Institute of Physical Medicine and Rehabilitation, Nancy, France \\ ${ }^{6}$ Laboratory for the Analysis of Posture, Equilibrium and Motor Function (LAPEM), University Hospital of Nancy, Vandoeuvre-lès-Nancy, France \\ 'Department of Otorhinolaryngology, Head and Neck Surgery, Inselspital, Bern University Hospital, University of Bern, Bern, Switzerland \\ ${ }^{8}$ Department of Pediatric Otolaryngology, University Hospital of Nancy, Nancy, France \\ ${ }^{9}$ European Institute for ORL-HNS, GZA Hospitals, Antwerp, Oosterveldlaan, Wilrijk, Belgium \\ ${ }^{10}$ Department of Oto-Rhino-Laryngology, Head and Neck Surgery, University Hospital, Grenoble Alpes, France \\ "Department of Otorhinolaryngology, University of Iceland, Reykjavík, Iceland \\ ${ }^{12}$ Department of Otorhinolaryngology, Akureyri Hospital, Akureyri, Iceland
}

ORCID IDs of the authors: A.M. 0000-0002-4499-3476; C.B. 0000-0003-3752-7650; G.D. 0000-0002-8311-0322; P.P. 0000-0002-4381-0850.

Cite this article as: Mallinson A, Maire R, Beyaert C, et al. Understanding and managing trauma induced vestibular deficits. J Int Adv Otol. 2021;17(6):559-565

OBJECTIVES: Traumatic brain injury occurs frequently worldwide. Half of traumatic brain injuries are related to falls or motor vehicle accidents. The term "concussion" is often used to describe a minor form of traumatic brain injury. These often involve decelerative events to the head (e.g., flexion/extension injury) and can also cause damage to the vestibular system of the inner ear.

MATERIALS AND METHODS: The European Society for Clinical Evaluation of Balance Disorders meets yearly and has proposed an investigation and analysis of the vestibular consequences of traumatic brain injury. This review paper outlines these discussions.

RESULTS: The Society discussed all aspects of trauma-induced vestibular disorders along with diagnosis and management. They also assessed the diagnostic tests available to investigate these disorders.

CONCLUSION: Trauma-induced vestibular disorders are difficult to manage, as our level of understanding of the pathology can be poor and anatomical localization can also be difficult. Accordingly, a definitive diagnosis cannot be pinpointed in many patients, but an extensive history taking is crucial to determine the nature and extent of vestibular involvement. Trauma can not only result in microtrauma to the central nervous system but can also significantly affect peripheral vestibular structures, particularly the otolith organs. The committee hopes that better understanding of trauma to the vestibular system, along with improvements in the field of radiology and vestibular assessments, will aid in more precise techniques of pinpointing pathology in order to develop an adapted treatment plan.

KEYWORDS: Balance disorders, clinical assessment, otoliths, vestibular trauma, whiplash

\section{INTRODUCTION}

Traumatic brain injury (TBI) is frequent worldwide and may lead to strong health and socio-economic problems. In the United States, about 1.6 million TBIs occur each year, corresponding to a rate of 540/100,000. ${ }^{1}$ The most frequent cause of TBI are falls (about 30\%), followed by motor vehicle accidents (20\%), direct hits by another person or object (this includes sports-related activities) $(18 \%)$, and assaults (10\%). ${ }^{2}$ Traumatic brain injury is more common in men than women and also in younger people. The degree of TBI is classified as mild, moderate, or severe in the Diagnostic and Statistical Manual of Mental Disorders, according to the 
duration of the loss of consciousness and post-traumatic amnesia, and the Glasgow Coma Scale at initial assessment. ${ }^{3}$ The term "concussion" corresponds to a minor form of TBI (mTBI) and both terms may be used synonymously. ${ }^{4}$ Minor form of TBls may account for as many as $80 \%$ of TBIs.

Trauma-induced disorders can often include decelerative events to the head such as traumatic flexion/extension injury or "whiplash" type of injury. Whiplash is a term used to describe the bony or soft tissue lesions resulting from sudden acceleration-deceleration forces applied to the neck and has been rated in 4 grades according to the severity of the injury. ${ }^{5}$

Sudden acceleration-deceleration events can also cause damage to the vestibular system of the inner ear. Patients suffering such damage often voice characteristic complaints. The exact nature of the mechanisms producing the vestibular symptoms is not yet clearly understood but may be related to alteration of the cervical somatosensory receptors or perhaps decelerative trauma to the otolithic structures of the ear. It is important to stress that vestibular injury (causing a recognized symptom set) from decelerative injury can occur regardless of whether an actual head strike occurred. Symptoms and signs often differ from classic vestibular presentations but in some patients can be persistent and debilitating. The most frequent vestibular complaints after trauma are benign paroxysmal positioning vertigo (BPPV, up to $38 \%$ ), which by definition is damage to the otoliths. Temporal bone fracture causing vestibular pathology (sometimes bilateral) can also occur. Perilymphatic fistula, post-traumatic Menière's disease, and central vestibular deficits must also be considered. In most cases, the vestibular symptoms disappear in a few weeks, but subtle symptoms can persist for months or years, accompanied by other persisting symptoms such as headache, fatigue, visual disturbance, cognitive and emotional impairments. This is often referred to as "post-concussion syndrome."6,7 Patients with persisting post-concussion symptoms are far less likely to return to work.

When a patient voices complaints after trauma (or at any time) suggesting vestibular involvement, it is crucial to take an indepth nonleading history and have the patient describe his or her symptoms in everyday language. Symptoms of vestibular injury are often referred to in the literature using terms such as "visually induced dizziness" or "persistent postural perceptual dizziness" (PPPD). Patients with symptoms of imbalance often voice persistent feelings of insecurity within their environment but often volunteer they are not noticeably off balance. Excessive and persistent nausea induced by minor activities is another recognized symptom of vestibular origin.

It remains quite a challenge to differentiate between peripheral inner ear dizziness and the dizziness associated with minimal TBI. If injury to the inner ear and the brain has occurred at the same time, they cannot be dissociated. However as mentioned, it is accepted that characteristic vestibular complaints after a concussion may be from damage to the inner ear.

The European Society for Clinical Evaluation of Balance Disorders aimed in one of its latest discussions to propose recommendations on the diagnosis and management of different types of trauma-induced vestibular disorders. This review paper outlines these discussions.

\section{Radiological Assessment}

When assessing the patient with vestibular complaints after head trauma, the presence of a fracture is an aspect that must be ruled out (or ruled in). Longitudinal and transverse fractures are the 2 main types of temporal bone fractures. ${ }^{8}$ Longitudinal fractures are most frequently seen and represent about $80 \%$ of temporal bone fractures. They usually result from temporo-parietal impacts to the head. Computed tomography scan imaging can reveal a linear fracture through the external auditory canal (EAC). The clinical manifestations include bleeding from the EAC, hematotympanum, and conductive hearing loss. Complications that can occur early or later on include secondary transitory facial weakness or paralysis ( $<25 \%$ of cases), otitis media (in cases of tympanic membrane perforation), and also meningitis (due to a cerebrospinal fluid fistula). Persistent conductive hearing loss can occur due to lesions of the ossicular chain such as dislocation of the incudostapedial joint with or without subluxation of the stapes. Other complications such as cholesteatoma (in case of tympanic laceration) or stenosis of EAC may appear some years later after the acute event.

Transverse fractures can also result from blows to the occiput. Computed tomography scan imaging reveals a linear break traversing the temporal bone perpendicular to the long axis of the petrous pyramid. Clinical manifestations are hematotympanum, and sometimes spontaneous nystagmus, which will beat toward the normal ear. Symptoms are a sudden cochleo-vestibular deficit with deafness and acute vertigo resulting from an ipsilateral peripheral vestibular loss. In about $50 \%$ of transverse fractures, the inner ear lesion is also accompanied by laceration of the facial nerve resulting in a permanent facial palsy. Because of the transverse linear break through the inner ear, the risk of meningitis or cerebrospinal fluid fistula is greater than in the case of longitudinal fractures. These types of fracture generate a complete loss of cochlear and vestibular function. Prognosis of facial palsy remains uncertain.

Malformations of the inner ear such as asymptomatic enlarged vestibular aqueduct, or dehiscence of the superior semicircular canal may become symptomatic after cranial trauma, even without fracture. In such situations, it is important to perform a CT scan.

Superficial siderosis of the central nervous system represents a late complication of cranial fractures which can also involve progressive unilateral or bilateral cochleo-vestibular deficits. ${ }^{9}$

\section{Functional Assessment}

It must be emphasized that there are no specific evaluations to document or quantify vestibular trauma related to acceleration-deceleration events. These patients are diagnosed with an extensive history, along with clinical and radiological assessment. As the vestibular system is a silent system, symptoms often include sensations of internal self motion, which is often accompanied by associated symptoms such as headache and photo/phonophobia. These patients often do not show any objective abnormalities in a clinical exam.

Clinical evaluation in an emergency ward to evaluate signs of acute vestibular impairment can often be helpful. However, vestibular assessments carried out at an early stage are often not helpful, partly because of the complexity of the vestibular system and also due to the lack of effective diagnostic testing. Unfortunately, this is quite 
often also the case for the long-term evaluation of residual vestibular symptoms, as the diagnosis remains elusive in $25 \%$ of post-traumatic vestibular disorders ${ }^{10}$ and because cervical involvement is difficult to assess.

Vestibulo-ocular Reflex Assessment Tools

The development of recent tests such as the skull vibration-induced nystagmus test ${ }^{11}$ and head impulse testing (HIT) can be particularly useful in the acute setting, as these tools can help to document a substantial peripheral vestibular deficit. However, in the presence of a suspected cervical injury, these must be used with caution in the emergency ward; head shaking test (HST) is not recommended until cervical spine pathology is ruled out radiologically (Table 1). Gaze testing, pursuit, and saccades should be performed to rule out a central lesion. Sometimes a spontaneous or positional nystagmus (DixHallpike maneuvre) under Frenzel goggles or skew deviation (using a cover test) can be observed. A spontaneous nystagmus is generally of peripheral origin when suppressed by visual fixation.

In the patient reporting neck pain in conjunction with spells of vertigo or unsteadiness, cervical examination must be carried out carefully, and further vascular investigations (cervical Doppler, Angio CT Scan, or Angio magnetic resonance imaging) are recommended.

\section{Vestibular-Evoked Myogenic Potential Testing}

Vestibular-evoked myogenic potential (VEMP) testing, which provides specific and sensitive otolithic assessments, is very useful and is able to detect abnormalities not shown by computerized dynamic posturography (CDP) or caloric testing. ${ }^{12}$ Its development as a clinical tool allowed for detailed and accurate evaluation and measurement of objective abnormalities, which are often bilateral. Documenting this is helpful as it has been shown that bilateral pathology is much more difficult to cope with (for the clinician and also the patient). ${ }^{13}$

Cervical Vestibular-Evoked Myogenic Potentials: The cervical VEMPs (CVEMPs) are primarily a saccular evaluation. They consist of analyzing the activity of the ipsilateral sternocleidomastoid muscle during neck flexion in response to an auditory stimulation. There are 3 different parameters of the response, which makes this a much more sensitive test than standard caloric testing. ${ }^{13}$
Ocular Vestibular-Evoked Myogenic Potentials: The ocular VEMPs (oVEMPs) are primarily utricular and are recorded from under the eyes. They are contralateral excitatory signals and are elicited by elevating the eyes to an angle of $30^{\circ}$ above midline. The oVEMP is a much more delicate signal, and its amplitude is a factor of 10 lower than standard cVEMP responses. Assessments are carried out in a fashion similar to the cVEMP protocol.

Another method of measuring utricular function is the subjective visual vertical (SVV) assessment. As mentioned, it is a subjective test but can still detect (and sometimes lateralize) abnormalities. One of the utricular tasks is to modulate ocular counterroll (i.e., perception of earth vertical). Utricular pathology can distort this perception, and this can be measured clinically by SVV.

\section{Postural Control Assessment}

One of the compounding issues in the vestibular trauma patient is the fact that some patients recover to a lesser degree than others who have suffered seemingly similar injuries. Patients with vestibular damage can always be expected to recover from their newly developed deficit, either via resolution or compensation. The concept of compensation involves effective use of alternative sensory inputs. However, some patients inexplicably do not compensate effectively for their vestibular loss. The whole process of compensation can itself be a challenge, as the alternate information sets which patients sometimes rely upon can themselves be orientationally disruptive (e.g., watching a train go past). Computerized dynamic posturography allows the clinician to assess a patient's performance when he or she is challenged with such disruptive stimuli. ${ }^{12}$

Posturography also has a secondary role. As mentioned previously, patients sometimes have symptoms which can sometimes override signs. This was identified some years ago in NASA research studying returning astronauts, whose symptoms are quite often debilitating. ${ }^{12}$ Symptoms are generated by the vestibular system in response to experiencing (and returning from) zero gravity situations. Patients often volunteer that this symptom complex, often referred to in the literature as "PPPD," is reproduced by the posturography assessment, and the literature strongly suggests that this is indicative of a vestibular deficit. ${ }^{12}$ This is extremely helpful not only to the assessor but also

Table 1. Tests Available for Vestibular Assessment. Frequency of Stimulation, Immobility Head vs Trunk (Keeping the Head in the Axis of the Trunk), and Vestibular Structures Involved

\begin{tabular}{|c|c|c|c|c|c|c|c|}
\hline \multirow{2}{*}{ Vestibular Test } & \multirow{2}{*}{ Frequency $(\mathrm{Hz})$} & \multirow{2}{*}{ Immobility Head/Trunk } & \multicolumn{5}{|c|}{ Vestibular Structures Involved } \\
\hline & & & Lateral SCC & Posterior SCC & Anterior SCC & Utricle & Saccule \\
\hline Caloric test & 0.003 & + & + & & & & \\
\hline Rotary chair & $0.01-0.05$ & + & + & & & & \\
\hline HST_Lateral & 2 & & & & & & \\
\hline HIT_Lateral & 6 & & + & & & & \\
\hline HIT-RALP & 6 & & & + & + & & \\
\hline HIT_LARP & 6 & & & + & + & & \\
\hline SVINT & 100 & + & + & & & + & \\
\hline oVEMP & 500 & + & & & & + & \\
\hline CVEMP & 700 & & & & & & + \\
\hline
\end{tabular}

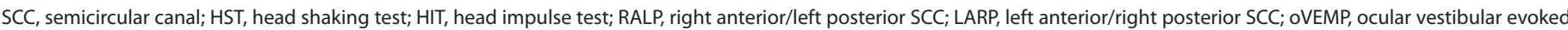
myogenic potential; cVEMP, cervical vestibular evoked myogenic potential. 
often a great relief to the patient who is often concerned that his or her complaints will be dismissed, as it is sometimes suggested that "since all the assessments are normal, there probably is not anything wrong with you."

Using virtual reality goggles, the visual system can also be challenged with movement information when the patient is standing on a posturography platform. In this way, postural control can be assessed in an orientationally inappropriate situation, that is, under situations of sensory conflict between visual input (movement) and vestibular input (lack of movement).

\section{Gait Assessment}

A growing number of studies over the past 2 decades have investigated the effects of TBI, in particular mild TBI or concussion, on gait. ${ }^{14}$ In an attempt to measure the ability to carry out the diverse set of activities required during daily ambulation, various experimental protocols of gait assessment have been developed. These include single-task simple gait, gait with simultaneous cognitive tasks (dualtask gait), and complex gait such as tandem gait, obstacle step-over tasks, or timed up-and-go test.

In healthy individuals, simple gait along a straight path is largely controlled through automatic subcortical locomotor processing requiring little executive control in healthy individuals. ${ }^{15,16}$ However dual-task gait and complex gait utilize frontal lobe executive functioning to facilitate the processing of simultaneous cognitive and motor demands. ${ }^{17}$ Fino et al $^{14}$ reviewed the effects of concussion on gait and showed slower single-task simple or complex gait during only the acute phase (0-10 days post-concussion), poor balance control (mediolateral movement of the body center of mass (COM)) in dual-task simple gait during the subacute phase (11-90 days post-concussion), and subacute gait abnormalities during specific complex gait tasks (e.g., slower tandem gait). A meta-analysis of studies in adults between 4 weeks and 6 months post-concussion found reduced maximal mediolateral COM displacement in dualtask gait. ${ }^{18}$

Concussion prognosis is a challenging clinical task that warrants early assessment (within 7-10 days of concussion) in order to identify the odds of developing "persistent post-concussion symptoms" (PPCS) (i.e., $\geq 28$ days post-concussion). It has been recently found that worse dual-task tandem gait test time and modified Balance Error Scoring System tandem stance performance will predict PPCS in pediatric patients, ${ }^{19}$ and greater dual-task transverse plane COM range of motion and lateral step variability will predict PPCS in young adult athletes. ${ }^{20}$

Patients who have a sustained TBI may report residual balance problems such as dizziness, unsteadiness, or imbalance which can linger on for months or even years. ${ }^{21}$ In people more than 3 months postconcussion, it was shown that symptom severity affects gait. In the study, it was shown that the concussion group walked and turned more slowly than normal subjects under single- and dual-task conditions, with less rhythm under dual-task gait. It was also shown that a higher Neurobehavioral Symptom Inventory (NSI) somatic/sensory subscore was related to higher single- and dual-task gait variability, and these patients also showed slower dual-task pacing and turning. ${ }^{22}$
In TBI, the most frequently reported balance impairments (especially in patients with vestibular disorders) involved sensory organization deficits between vestibular, visual, and somatosensory systems ${ }^{23}$ and possibly cervical somatosensory disorders. ${ }^{24}$ Patients also reported impairments of their gait "not being automatic." This has been attributed to damage in brain regions responsible for executive function. ${ }^{25}$ Although gait ataxia has been well related to vestibular dysfunction in patients with moderate-to-severe $\mathrm{TBI}$, the nature of balance problems in chronic mild TBI may be related to impairment of the "automatic gait" pathways. ${ }^{26}$ It has been shown in chronic mild TBI patients who voiced complaints of "feeling dizzy" and "loss of balance" on the NSI that dual-task gait cost was negatively associated with the Dizziness Handicap Inventory, whereas the Sensory Organization Test vestibular score failed to predict balance-related disability. ${ }^{26}$

\section{Vestibular Trauma and Electroencephalography}

Concussion can of course affect the brain but, as discussed previously, can also impact the ear; this can be mediated through pressure in the vestibular aqueduct affecting the membranous labyrinth. Although it is rare to have purely vestibular symptoms, patients' complaints can range from mild disequilibrium to rotational vertigo in any plane.

Although electroencephalography (EEG) can be useful in assessing trauma, it is important to note that EEG abnormalities do not relate to severity of complaints in mTBI, and therefore, it is not helpful in differentiating groups or determining anatomical location. However, EEG may be a helpful tool in helping to pinpoint or rule out central pathology.

Electroencephalography was the first clinical neuro-diagnostic assessment that revealed abnormal brain function following TBI. Quantitative EEG (qEEG) recordings can be used to demonstrate several quantitative trends. Among these, spectral analysis and coherence are of particular interest in $\mathrm{mTBI}$. In the acute stage, qEEG usually shows immediate reduction in mean alpha frequency, with increased theta, increased delta activity, and increased theta/alpha ratio. ${ }^{27}$

The reduced alpha and increased delta frequency noted above can persist for weeks to months, but there is a 1-2 Hz increase in the frequency of the mean alpha frequency; this is a sign of recovery to the original baseline from the post-traumatic slowing. The majority of acute qEEG abnormalities resolve by 3 months, and $90 \%$ resolve within 1 year of the head trauma. ${ }^{28}$ Regardless of whether the abnormalities are subtle or overt, most post-traumatic qEEGs show evidence of improvement over time. ${ }^{29}$

As the evidence for the cortical representation of the vestibular system grows, so does the evidence for vestibular symptoms occurring as a manifestation of associated focal cortical epileptic activity. Other additional symptoms are nausea or vomiting, tinnitus, ipsilateral or contralateral paresthesia, olfactory and gustatory hallucinations, as well as depersonalization. A diagnosis is strongly supported by positive EEG findings where abnormalities over the parietal or temporoparietal-occipital areas are prominent. ${ }^{30}$

\section{Medical Legal Assessment of Vestibular Complaints}

In the medical legal setting, there is often the need to provide evidence of the effects of injuries or their sequelae, in particular following whiplash injuries, in the context of insurance claims, work stoppages, or compensation claims, especially in cases which result in court 
proceedings. However, this is sometimes a challenge, as patients with complaints of a vestibular nature following trauma (usually a whiplash type of injury) may sometimes not have objective signs, but just a history strongly suggestive of vestibular involvement. These patients often describe similar symptom sets to those who had suffered cervical deceleration injury without an actual head blow, suggesting perhaps that dizziness reported after mTBI may be from the inner ear. ${ }^{31}$

Initially, investigation consisted of using videonystagmography (VNG), where $15-20 \%$ had abnormalities, and CDP, where approximately 50\% of patients with persistent post-traumatic vestibular symptoms had measured balance abnormalities. Posturography is a more general test for overall balance. However, test abnormalities are able to legitimize complaints that are often dismissed as psychiatric by multiple other physicians. A helpful aspect of CDP is that the sensation induced during assessment is frequently volunteered as simulating their symptom set. ${ }^{32}$ This is a separate useful piece of information, suggesting vestibular pathology, and should be asked about if not spontaneously declared.

The advent of VEMP testing has allowed for detailed measurement of otolithic function. Despite prolonged vestibular physiotherapy, many patients often remain symptomatic. Vestibular-evoked myogenic potentials are able to detect abnormalities not shown by CDP, and studies have shown that deficits are quite common and often bilateral. ${ }^{13}$ This can aid in determining the final symptomatic status of a patient. Unfortunately, some patients have complaints which are persistent and likely permanent; this is again an important factor in the medical legal setting.

Cervical vertigo is a conundrum. Pre-existing conditions such as basilar impression and cervical degenerative disorders can compound this. Any vestibular complaints may be a compound of pre-existing disorders combined with degenerative factors and the acute event (whether it is traumatic or nontraumatic). Again the situation can be clarified with an in-depth history taking.

One factor in assessment of the medical legal patient, especially when using posturography, is to address "discordances" (defined as "lack of agreement") in clinical assessment. ${ }^{32}$ Sometimes there is disagreement between a clinical assessment and measured abnormalities; sometimes the results within the assessment do not agree. This may be thought of as "malingering" or an attempt to exaggerate what is wrong, but this is not always the case. For example, performance on more difficult trials may sometimes be better than on simpler trials. This can be suggestive of malingering but in some situations can be a legitimate finding. The extreme malingerer and the genuine patient are at opposite ends of a spectrum, but there are many variations along this spectrum, and clinicians need to be cautious, as a posturography assessment may or may not be diagnostically helpful.

In brief, interpretation of posturography performance can at times be difficult, and a patient's results must be correlated with clinical findings by an unbiased clinician and without stereotyping the patient. It is only in this fashion that assessment in a diagnostic setting can be carried out in an appropriate manner.

\section{Vestibular Effects of Barotrauma}

Barotrauma is defined as physical tissue damage due to failure of equalization of pressure of the internal ear with that of the surrounding environment. Compressive or expansive forces and shear to overstretched tissues can lead to damage in the middle or inner ear. Perforation of the tympanic membrane (TM) and rupture of the oval and/or round window membrane can also occur. ${ }^{33}$ Most examples of ear barotrauma occur in diving or in air travel, although there are other causes discussed in the literature, such as mountain climbing, skiing or explosive blast. ${ }^{34-36}$ A TM rupture could lead to caloric stimulation of the vestibular apparatus, vertigo, and disequilibrium under water, which is a highly dangerous situation in combination with an (unexperienced) panicking diver. ${ }^{33}$ Tympanic membrane rupture can occur at depths as shallow as $1.2 \mathrm{~m}^{37}$

More than $80 \%$ of all diving complications occur in the head and neck, $65 \%$ of these complications involving the ear. ${ }^{38}$ Diving injuries tend to be correlated with advancing age, alcohol usage, obesity, asthma and chronic obstructive pulmonary disease, chronic sinusitis, and otitis. ${ }^{39}$

Inner ear trauma can also occur from pressure changes in the middle ear, ${ }^{33}$ but the inner ear can also suffer (e.g., perilymphatic fistula). ${ }^{40}$ When the pressure differential reaches a limit of $>90 \mathrm{mmHg}$ (or a pressure equivalent of 10 feet of seawater), the Eustachian tube will close and a successful Valsalva maneuver will be prevented. ${ }^{40}$ When the diver exerts repeatedly forceful Valsalva maneuvers in this condition, due to the urge coming from the feeling of an increased ear pressure, the intracranial pressure increases accordingly. This will be transmitted to the cochlea via the perilymphatic duct, causing an increase in the perilymphatic fluid and eventually an explosive rupture of the round window. ${ }^{41}$ On the other hand, when a Valsalva maneuver is being performed successfully (i.e., when the Eustachian tube is successfully opened), there is a sudden increase in middle ear pressure, causing the TM to bulge rapidly outward. This causes the ME bones and the oval window to be pulled away as well, creating a decrease in perilymphatic pressure in the cochlea, which can cause an implosive rupture of the oval or round window, cochlear haemorrhage, or a rupturing of one of the cochlear membranes. . $^{33,42}$

Typically, barotrauma patients have a combination of vestibular and hearing deficits with complaints of vertigo, ${ }^{42}$ but in some cases, only suffer a sensorineural hearing loss. The barotrauma that a diver might experience during descent can impair middle ear ventilation during ascent, leading to a progression of barotrauma. ${ }^{33}$

Surgical exploration is sometimes necessary in case of a ruptured TM or suspected perilymphatic fistula, with patching of the round or oval window if necessary. ${ }^{43}$

\section{Vestibular Consequences of Head Trauma in Children}

In case of sudden trauma-induced vestibular dysfunction in children, the process of central compensation is probably more effective. Because of reduced ability in younger children to describe their symptoms, it is more difficult to identify mild symptoms which may be more subtle. Objective evaluations by parents are often useful in this regard. Vestibular assessment, especially in the younger child, may also be more of a challenge to perform and analyze, and the whole process sometimes needs to be carried out in the setting of an apprehensive patient (and parents). Given these factors, accurate vestibular assessments are especially difficult and the presence of pathology is likely underestimated in the pediatric population. 
In the pediatric population, diagnosis of BPPV must always be kept in mind. Children comprise only about $1 \%$ of all BPPV patients, and almost all pediatric cases result from head trauma.

Two other possibilities must be always kept in mind, otic capsule bone fracture and perilymphatic fistula. In both cases, pneumoccocal vaccination is required, and also, surgical closure of the leakage must be undertaken as soon as possible to avoid infectious complications (which will always occur) and hearing loss (which will occur sometimes).

In severe head trauma, many overlapping factors (i.e., associated lesions) in different locations can make pathophysiological analysis extremely complex in children (as well as in adults). Potential vestibular sequelae, in this case, take part in daily life difficulties (schooling, sports, etc.) and then must be considered. These challenges will be amplified in the presence of bilateral pathology. In short, in all children, even extremely young ones, 2 points should be kept in mind: in case of head trauma, the possibility of vestibular involvement should be explored, and a history of head trauma (even remotely) should be asked about in all vestibular dysfunction presentations.

\section{Vestibular Consequences of Head Trauma in Sport}

The sports most associated with mild TBI are boxing (involving knockouts), team sports (soccer, American football, rugby, ice hockey, water polo), ${ }^{44,45}$ mountain biking, ${ }^{46}$ and skiing, especially freestyle (halfpipe).

In boxing, a knockout (which results from a cerebral concussion and/ or loss of ability to maintain posture) is defined as an athlete not being able to restart the fight after 10 seconds. The impact, often involving sudden rotation and extension of the head backward, can cause a cerebral trauma and an impairment of the centres ensuring muscular tone and balance. This can result in a loss of integration of vestibular, visual, and proprioceptive (cervical muscles) information that are under cerebellar control, generating a sudden interruption of postural tone.

A soccer player can "head a ball" (i.e., be hit in the head) several dozen times in each game. While these blows can reach several dozen times the gravitational force $\left(9.8 \mathrm{~m} / \mathrm{s}^{2}\right)$ without causing a concussion, the consequences of their repetition can be documented, depending on the sensitivity of the clinical tests. ${ }^{47}$ Shock data can be collected by head impact telemetry sensors with gyroscopes and accelerometers (documenting number of hits during the match or training, their strength and direction). Head injury can be influenced by acceleration, change in momentum, and duration of impact. ${ }^{48}$

The accumulation of impacts (crashes, vibrations) during the career of a downhill mountain biker may generate micro-trauma to the central nervous system and/or peripheral vestibular structures, particularly the otoliths. ${ }^{46}$

Concussions are more common among adolescents than in other age groups, particularly because sports participation is more common in this age group. ${ }^{49}$ It is necessary to recognize acute emergencies for the sportsman and the possibility of acute trauma to the labyrinth. This is important because it may be necessary to stop competition and training.

The danger of concussions may be linked to their repetition. The treatment, depending on the etiology, is physiotherapy (stabilization of the gaze, adaptation, habituation, liberatory maneuvres, postural and walking exercises), pharmacological and/or surgical. Prevention (helmet protection, dodging, strengthening of the cervical musculature) should be adapted according to the type of sport.

Management guidelines concerning return to play issues have been published, in particular with regard to the type of trauma, age (school-aged children through adulthood), and type of sport. ${ }^{50}$

\section{CONCLUSION}

Diagnosis of peripheral vestibular involvement in head trauma should always be explored so that the patient can be managed and treated appropriately. Vestibular assessment is sometimes, but not always helpful, and unfortunately, a definitive diagnosis cannot be pinpointed in up to $25 \%$ of patients. History taking is the most important aspect of investigating these patients. Identifying a patient who has suffered vestibular trauma is important from the management point of view. This is because rest and inactivity is often advised for post-traumatic injury patients, but this is contraindicated in the patient who has suffered a vestibular insult, as they may be required to compensate for a persistent vestibular deficit. An inappropriate diagnosis (or "label") could possibly impede a rehabilitative plan.

Vestibular assessments are occasionally but not frequently helpful in documenting peripheral damage in the acute setting, but it must be emphasized that negative tests do not rule out pathology. A cervical or central origin can sometimes be documented by ocular motor abnormalities. Posturography and virtual reality are often helpful not only in documenting but also in reproducing a patient's symptoms, which can also aid the clinician at furthering the understanding of the presenting complaints.

Peer Review: Externally peer-reviewed.

Author Contributions: Concept - A.M., R.M., C.B., D.V., L.C., N.L., R.V., G.D., H.P., P.P.; Design - A.M., R.M., C.B., D.V., L.C., N.L., R.V., G.D., H.P., P.P.; Supervision A.M., R.M., C.B., D.V., L.C., N.L., R.V., G.D., H.P., P.P.; Resource - A.M., R.M., C.B., D.V., L.C., N.L., R.V., G.D., H.P., P.P.; Materials - A.M., R.M., C.B., D.V., L.C., N.L., R.V., G.D., H.P., P.P.; Data Collection and/or Processing - A.M., R.M., C.B., D.V., L.C., N.L., R.V., G.D., H.P., P.P.; Analysis and/or Interpretation - A.M., R.M., C.B., D.V., L.C., N.L., R.V., G.D., H.P., P.P.; Literature Search - A.M., R.M., C.B., D.V., L.C., N.L., R.V., G.D., H.P., P.P.; Writing - A.M., R.M., C.B., D.V., L.C., N.L., R.V., G.D., H.P., P.P.; Critical Reviews - A.M., R.M., C.B., D.V., L.C., N.L., R.V., G.D., H.P., P.P.

Acknowledgments: Thanks to the University of Lorraine for assistance in staging the conference.

Conflict of Interest: The authors have no conflict of interest to declare.

Financial Disclosure: The authors declared that this study has received no financial support.

\section{REFERENCES}

1. Rutland-Brown W, Langlois JA, Thomas KE, Xi YL. Incidence of traumatic brain injury in the United States, 2003. J Head Trauma Rehabil. 2006;21(6):544-548. [CrossRef]

2. Langlois JA, Rutland-Brown W, Wald MM. The epidemiology and impact of traumatic brain injury: a brief overview. I Head Trauma Rehabil. 2006;21(5):375-378. [CrossRef]

3. American Psychiatric Association. Diagnostic and Statistical Manual of Mental Disorders (DSM-5), Virginia: American Psychiatric Association; 2013:626. 
4. Ruff RM, Iverson GL, Barth JT, Bush SS, Broshek DK, NAN Policy and Planning Committee. Recommendations for diagnosing a mild traumatic brain injury: a National Academy of Neuropsychology education paper. Arch Clin Neuropsychol. 2009;24(1):3-10. [CrossRef]

5. Spitzer WO, Skovron ML, Salmi LR, Cassidy JD, et al. Scientific monograph of the Quebec Task Force on whiplash associated disorders: redefining whiplash and its management. Spine. 1995;20(suppl 8):1-73S

6. Ryan LM, Warden DL. Post concussion syndrome. Int Rev Psychiatry. 2003;15(4):310-316. [CrossRef]

7. Chong CS. Management strategies for post-concussion syndrome after mild head injury: a systematic review. HKJ Occup Ther. 2008;18(2):59-67. [CrossRef]

8. Schuknecht HF. Pathology of the Ear. 2nd ed. Philadelphian: Lea \& Febiger; 1993:279-281.

9. Wagner F, Buchwalder M, Wiest R, Caversaccio MD, Vibert D. Superficial siderosis of the central nervous system: neurotological findings related to magnetic resonance imaging. Otol Neurotol. 2019;40(1):31-37. [CrossRef]

10. Marcus $H J$, Paine $H$, Sargeant $M$, et al. Vestibular dysfunction in acute traumatic brain injury. J Neurol. 2019;266(10):2430-2433. [CrossRef]

11. Dumas G, Curthoys IS, Lion A, Perrin P, Schmerber S. The skull vibrationinduced nystagmus test of vestibular function: a review. Front Neurol. 2017;8:41. [CrossRef]

12. Mallinson Al, Kuijpers ACM, Van Zwieten G, Kakal J, Mullings W, Longridge NS. Computerized dynamic posturography does not detect measured CVEMP and OVEMP abnormalities. Gait Posture. 2019;67:248250. [CrossRef]

13. Longridge NS, Mallinson Al. Tone induced cervical and ocular vestibularevoked myogenic potentials: comparing abnormalities in traumatic and non-traumatic vestibular disease. J Laryngol Otol. 2018;132(10):906-910. [CrossRef]

14. Fino PC, Parrington $L$, Pitt $W$, et al. Detecting gait abnormalities after concussion or mild traumatic brain injury: a systematic review of single-task, dual-task, and complex gait. Gait Posture. 2018;62:157-166. [CrossRef]

15. Maire R, Gauchard GC, Deviterne D, Magnusson M, Kingma H, Perrin PP. European Society for Clinical Evaluation of Balance Disorders: discussion about dual-task conditions combining postural control with cognitive tasks. Laryngoscope. 2010;120(10):2108-2109. [CrossRef]

16. Clark DJ. Automaticity of walking: functional significance, mechanisms, measurement and rehabilitation strategies. Front Hum Neurosci. 2015;9:246. [CrossRef]

17. Clark DJ, Rose DK, Ring SA, Porges EC. Utilization of central nervous system resources for preparation and performance of complex walking tasks in older adults. Front Aging Neurosci. 2014;6:217. [CrossRef]

18. Galea OA, Cottrell MA, Treleaven JM, O'Leary SP. Sensorimotor and physiological indicators of impairment in mild traumatic brain injury: a metaanalysis. Neurorehabil Neural Repair. 2018;32(2):115-128. [CrossRef]

19. Van Deventer KA, Seehusen CN, Walker GA, Wilson JC, Howell DR. The diagnostic and prognostic utility of the dual-task tandem gait test for pediatric concussion. J Sport Health Sci. 2021;10(2):131-137. [CrossRef]

20. Howell DR, Mayer AR, Master CL, et al. Prognosis for persistent post concussion symptoms using a multifaceted objective gait and balance assessment approach. Gait Posture. 2020;79:53-59. [CrossRef]

21. Kleffelgaard I, Roe C, Soberg HL, Bergland A. Associations among selfreported balance problems, post-concussion symptoms and performance-based tests: a longitudinal follow-up study. Disabil Rehabil. 2012;34(9):788-794. [CrossRef]

22. Martini DN, Parrington L, Stuart S, Fino PC, King LA. Gait performance in people with symptomatic, chronic mild traumatic brain injury. J Neurotrauma. 2021;38(2):218-224. [CrossRef]

23. Kolev OI, Sergeeva M. Vestibular disorders following different types of head and neck trauma. Funct Neurol. 2016;31(2):75-80. [CrossRef]

24. Brandt T, Bronstein AM. Cervical vertigo. J Neurol Neurosurg Psychiatry. 2001;71(1):8-12. [CrossRef]

25. McCulloch KL, Buxton E, Hackney J, Lowers S. Balance, attention, and dual-task performance during walking after brain injury: associations with falls history. J Head Trauma Rehabil. 2010;25(3):155-163. [CrossRef]
26. Joseph AC, Lippa SM, Moore B, et al. Relating self-reported balance complaints to sensory organization and dual-tasking in chronic traumatic brain injury. Phys Med Rehabil. 2020;13(8):870-879. [CrossRef]

27. Schmitt S, Dichter MA. Electrophysiologic recordings in traumatic brain injury. In: Grafman J, Salazar AM, eds. Traumatic Brain Injury, Part l; Handbook of Clinical Neurology. Vol 127 (3rd series). Amsterdam, Netherlands: Elsevier; 2015:319-339.

28. Haneef Z, Levin HS, Frost JD, Mizrahi EM. Electroencephalography and quantitative electroencephalography in mild traumatic brain injury. J Neurotrauma. 2013;30(8):653-656. [CrossRef]

29. Rapp PE, Keyser DO, Albano A, et al. Traumatic brain injury detection using electrophysiological methods. Front Hum Neurosci. 2015;9:11. [CrossRef]

30. Hewett R, Bartolomei F. Epilepsy and the cortical vestibular system: tales of dizziness and recent concepts. Front Integr Neurosci. 2013;7:73. [CrossRef]

31. Longridge NS, Mallinson AI. Visual vestibular mismatch in work-related vestibular injury. Otol Neurotol. 2005;26(4):691-694. [CrossRef]

32. Perrin $P$, Mallinson A, Van Nechel C, et al. Defining clinical-posturographic and intra-posturographic discordances: what do these two concepts mean? J Int Adv Otol. 2018;14(1):127-129. [CrossRef]

33. Livingstone DM, Smith KA, Lange B. Scuba diving and otology: a systematic review with recommendations on diagnosis, treatment and postoperative care. Diving Hyperb Med. 2017;47(2):97-109. [CrossRef]

34. Farmer JC Jr. Diving injuries to the inner ear. Ann Otol Rhinol Laryngol Suppl. 1977 Jan-Feb;86(1 Pt 3 Suppl 36):1-20.

35. FeeGA.Traumatic perilymphatic fistulas.ArchOtolaryngol.1968;88(5):477480. [CrossRef]

36. Akin FW, Murnane OD, Hall CD, Riska KM. Vestibular consequences of mild traumatic brain injury and blast exposure: a review. Brain Inj. 2017;31(9):1188-1194. [CrossRef]

37. Becker GD, Parell GJ. Barotrauma of the ears and sinuses after scuba diving. Eur Arch Otorhinolaryngol. 2001;258(4):159-163. [CrossRef]

38. Roydhouse N. 1001 disorders of the ear, nose and sinuses in scuba divers. Can J App/ Sport Sci. 1985;10(2):99-103.

39. Battisti AS, Haftel A, Murphy-Lavoie HM. Barotrauma. In: StatPearls [Internet]. Treasure Island, FL: StatPearls Publishing; 2020.

40. Shupak A, Doweck I, Greenberg E, et al. Diving-related inner ear injuries. Laryngoscope. 1991;101(2):173-179. [CrossRef]

41. Dumas G, Perrin P. Ear and aquatic sports. Sci Sports. 2001;16(5):263-271. [CrossRef]

42. Rozycki SW, Brown MJ, Camacho M. Inner ear barotrauma in divers: an evidence-based tool for evaluation and treatment. Diving Hyperb Med. 2018;48(3):186-193. [CrossRef]

43. Klingmann C, Praetorius M, Böhm F, Tetzlaff K, Plinkert PK. Fitness to dive in the otorhinolaryngological field. HNO. 2008;56(5):509-518. [CrossRef]

44. Blumenfeld RS, Winsell JC, Hicks JW, Small SL. The epidemiology of sports-related head injury and concussion in water polo. Front Neurol. 2016;7:98. [CrossRef]

45. Hunter LE, Branch CA, Lipton ML. The neurobiological effects of repetitive head impacts in collision sports. Neurobiol Dis. 2019;123:122-126. [CrossRef]

46. Lion A, Vibert D, Bosser G, Gauchard GC, Perrin PP. Vertigo in downhill mountain biking and road cycling. Eur J Sport Sci. 2016;16(1):135-140. [CrossRef]

47. Hwang S, Ma L, Kawata K, Tierney R, Jeka JJ. Vestibular dysfunction after subconcussive head impact. J Neurotrauma. 2017;34(1):8-15. [CrossRef]

48. O'Connor KL, Rowson S, Duma SM, Broglio SP. Head-impact-measurement devices: a systematic review. J Athl Train. 2017;52(3):206-227. [CrossRef]

49. Loosemore $\mathrm{M}$, Knowles $\mathrm{CH}$, Whyte GP. Amateur boxing and risk of chronic traumatic brain injury: systematic review of observational studies. Br J Sports Med. 2008;42(11):564-567. [CrossRef]

50. Silverberg ND, laccarino MA, Panenka WJ, et al. Management of concussion and mild traumatic brain injury: a synthesis of practice guidelines. Arch Phys Med Rehabil. 2020;101(2):382-393. [CrossRef] 